\title{
Laser Treatment of Acne Vulgaris
}

\author{
Ming H. Jih, M.D., Ph.D., ${ }^{1}$ and Arash Kimyai-Asadi, M.D. ${ }^{1}$
}

Traditional medical treatments for acne vulgaris include a variety of topical and oral medications. The combination of poor compliance, lack of durable remission, and potential side effects are common drawbacks to these treatments. The use of lasers and light devices has increased dramatically in recent years due to the overall ease of treatment, predictable clinical efficacy, and minimal adverse effects. A variety of light and laser devices has been used for the treatment of acne, including the potassium titanyl phosphate (KTP) laser, the 585- and 595-nm pulsed dye lasers, the 1450-nm diode laser, radiofrequency devices, intense pulsed light sources, low-intensity light treatment, and photodynamic therapy using 5-aminolevulinic acid and indocyanine green. These devices are thought to target underlying pathogenic factors such as Propionibacterium acnes colonization, increased sebaceous gland activity, and the cutaneous inflammatory response. Lasers in particular also have a central role in the management of acne scarring, which tends to be refractory to medical therapies. Fractional photothermolysis, the $1450-\mathrm{nm}$ diode laser, and pulsed dye lasers have been used in the nonablative treatment of acne scars with significant success. In this article, we review the current status of light- and laser-based treatment of acne and related conditions and briefly review the use of lasers for the treatment of acne scarring.

KEYWORDS: Acne vulgaris, laser, photodynamic therapy, acne scarring

Acne vulgaris is one of the most common dermatologic conditions and affects the vast majority of people at some point during their lifetime. ${ }^{1}$ The pathogenesis of acne appears to be multifactorial. Abnormal differentiation and desquamation of follicular keratinocytes result in the formation of microcomedones; however, the formation of actual inflammatory acne lesions appears to depend on the proliferation of Propionibacterium acnes (P. acnes) bacteria in the microcomedones and the metabolization of trapped sebum into proinflammatory free fatty acids.

The treatment of mild acne includes various topical antimicrobials, retinoids, and keratolytics used alone or in combination. These topical modalities require frequent application by the patient and may result in clinically significant skin irritation. Moderate inflammatory acne requires the long-term use of oral antibiotics, which may be associated with increased bacterial resistance. More severe nodulocystic acne or acne resulting in significant dyspigmentation or scarring may require systemic isotretinoin. Isotretinoin is known to be associated with several serious side effects including teratogenicity, requires careful laboratory monitoring, and, more recently, mandatory registration. Many patients require continuous treatment with topical and oral medications for months or years, and compliance with treatment often becomes a major issue. Moreover, even with consistent use of individual and combination therapies, patients often continue to develop new acne lesions for years.

\footnotetext{
${ }^{1}$ DermSurgery Associates, Houston, Texas.

Address for correspondence and reprint requests: Ming H. Jih, M.D., Ph.D., DermSurgery Associates, 7515 Main, Suite 240, Houston, TX 77030.

Lasers in Plastic Surgery; Guest Editor, Ramsey F. Markus, M.D.
}

Semin Plast Surg 2007;21:167-174. Copyright (C) 2007 by Thieme Medical Publishers, Inc., 333 Seventh Avenue, New York, NY 10001, USA. Tel: $+1(212)$ 584-4662.

DOI 10.1055/s-2007-991185. ISSN 1535-2188. 
Light- and laser-based treatment options have, in recent years, become established as an alternative to traditional topical and oral medications. Lights and lasers appear to reduce inflammatory acne lesions by targeting some of the main pathophysiologic factors such as $P$. acnes bacteria, sebaceous gland activity, and by reducing inflammation. The initial therapeutic use of light sources to treat acne relied on the endogenous porphyrins produced by $P$. acnes, however, this was limited by the small amount of endogenous porphyrins naturally produced. Augmented bactericidal activity subsequently became possible with the addition of exogenous porphyrin precursors, such as aminolevulinic acid. More recently, various lasers, intense pulsed light, and radiofrequency devices have been used for the treatment of inflammatory acne vulgaris. Some of the laser devices provide simultaneous improvements in both inflammatory acne as well as scarring caused by acne. However, laser and light sources do not appear to be very effective for the treatment of noninflammatory comedonal acne.

\section{LASER, LIGHT SOURCE, AND RADIOFREQUENCY TREATMENT OF ACNE}

\section{2-nm Potassium Titanyl Phosphate Laser}

The potassium titanyl phosphate (KTP) vascular laser has generally been used for the treatment of telangiectases and rosacea but has also recently been shown to be effective for the treatment of acne. Although the exact mechanism of action is unclear, selective photothermolysis of blood vessels or a photodynamic effect of the laser on $P$. acnes and/or sebaceous glands have all been postulated. In a split face study of 26 patients with a KTP laser (Aura; Laserscope, San Jose, CA), a 34.9\% and $20.7 \%$ reduction in acne severity was achieved at 1 week and 4 weeks after four treatments. ${ }^{2}$ In another study, 25 patients who were treated with the KTP laser (Aura) at fluences ranging from 6 to $12 \mathrm{~J} / \mathrm{cm}^{2}$ achieved 60 to $70 \%$ clearing after six treatments. ${ }^{3}$

\section{5-nm and 595-nm Pulsed Dye Laser}

As with the KTP laser, the pulsed dye laser has been used mainly for the selective photothermolysis of vascular disorders, as well as other inflammatory disorders such as psoriasis and telangiectatic rosacea. In addition, the pulsed dye laser is widely known to stimulate dermal remodeling and collagen production. Thus, it has been used for the treatment of hypertrophic scar, keloids, as well as, acne scarring. ${ }^{4}$ Recent studies have shown that when used at low, nonpurpuric fluences, this laser can also reduce inflammatory acne. The effect of this laser on acne improvement was believed to be mediated by decreasing P. acnes or sebaceous gland activity; however, a recent study found the pulsed dye laser to have no effect on the degree of $P$. acnes colonization or sebum production as measured by the standardized application of absorptive tape. ${ }^{5}$ Rather, this study found a significant upregulation of transforming growth factor $\beta$, which is a potent stimulator of neocollagenesis and a potent inhibitor of inflammation. Thus, the efficacy of this laser on inflammatory acne is likely through its local antiinflammatory effects.

There have been several studies on the clinical efficacy of this laser for inflammatory acne. In a randomized controlled trial, 41 adults were assigned to receive either a single low-fluence $\left(1.5\right.$ or $3.0 \mathrm{~J} / \mathrm{cm}^{2}, 350$-microsecond pulse duration) treatment with the $585-\mathrm{nm}$ pulsed dye laser $(n=31)$ or a placebo sham treatment $(n=10)$. Patients were followed for 12 weeks, at which point the average total lesion count fell by $53 \%$ in patients treated with the laser compared with $9 \%$ in the controls. Similarly, the inflammatory lesion counts fell by $49 \%$ in pulsed dye laser patients compared with $10 \%$ in controls. ${ }^{6}$ However, a separate split-face study of 40 patients failed to show any statistically significant reductions in acne lesions after one or two treatments with the $585-\mathrm{nm}$ pulsed dye laser at a fluence of $3 \mathrm{~J} / \mathrm{cm}^{2}$ and 350-microsecond pulse duration. ${ }^{7}$

The 595-nm pulsed dye laser (VBeam; Candela Corp.) has also been used in conjunction with the 1450-nm diode laser (Smoothbeam; Candela Corp., Wayland, MA) to treat both acne vulgaris and postinflammatory erythema resulting from acne. In one noncontrolled study of 15 patients conducted in our practice, the lesions counts dropped by a mean of $52 \%$, $63 \%$, and $84 \%$ after 1,2 , and 3 treatments, respectively $(p<0.01) .{ }^{8}$ In addition to improvements in acne and acne scarring, significant improvement in post-inflammatory erythema was also noted and may be attributed to the selective photothermolysis of vessels by the pulsed dye laser. Although the combination of the two lasers may provide better targeting of different pathophysiologic contributors to acne, it is difficult to know whether the improvement is greater than that achieved with either the pulsed dye laser or the 1450-nm diode laser alone.

\section{0-nm Diode Laser}

Treatment with the infrared $1450-\mathrm{nm}$ diode laser (Smoothbeam; Candela Corp., Wayland, MA) with a dynamic cooling device has been shown to safely and effectively reduce inflammatory acne lesions of the face with fluences as high as $14 \mathrm{~J} / \mathrm{cm}^{2}$. Because of its efficacy, this laser has become a common clinical modality in the laser treatment of acne.

At a high fluence of $24 \mathrm{~J} / \mathrm{cm}^{2}$ in a rabbit ear model, this device has been shown to cause thermal coagulation of the sebaceous lobule and associated hair follicle through peak thermal heating of the upper to mid dermis up to a depth of $500 \mu \mathrm{m} .{ }^{9}$ Although such a high 
degree of thermal injury and coagulation has not been observed at fluences lower than or equaling $14 \mathrm{~J} / \mathrm{cm}^{2}$ as used in clinical practice, the presumed mechanism of acne improvement is through heating of the sebaceous gland and associated structures. It is believed that this heating of the sebaceous gland results in reduced sebaceous gland activity that subsequently leads to a reduction in inflammatory acne lesions. The initial study of the clinical efficacy of this laser on acne was performed by treating acne on the back. A significant reduction in lesion count was found in the treated side compared with the control side. ${ }^{9}$

The first report of the efficacy of this laser in the treatment of facial acne was an uncontrolled pilot study conducted by our practice. Nineteen patients with inflammatory acne, many of whom were refractory to traditional medical treatment, underwent three treatments at 4- to 6-week intervals using a fluence of $14 \mathrm{~J} / \mathrm{cm}^{2}$. The decrease in lesion counts from baseline was $37 \%$ after the first treatment, $58 \%$ after the second treatment, and $83 \%$ after the third treatment. Pain was well tolerated, and adverse effects were limited to transient erythema and edema at treatment sites. ${ }^{10}$

A subsequent study conducted by our practice on 20 patients who had received treatment with this laser found that increasing the fluence to $16 \mathrm{~J} / \mathrm{cm}^{2}$ using a prototype unit capable of achieving higher fluences did not provide improved results compared with $14 \mathrm{~J} / \mathrm{cm}^{2} .{ }^{11}$ More importantly, this study was the first to evaluate long-term improvement in inflammatory acne after cessation of laser treatments, as one of the significant criticisms of laser treatment prior to this study was that the sustained long-term efficacy of laser treatments had not been established. In this study, a reduction of $76.1 \%$ from baseline was sustained 12 months after the third and final laser treatment (Fig. 1). Thus, this study helped demonstrate that the 1450-nm diode laser provides a long-term remission in acne, a property not commonly seen with either topical medications or oral antibiotics but which is more commonly associated with oral isotretinoin. This study also demonstrated a significant improvement in acne scarring (Fig. 1). Most of the improvement in acne scarring developed over the 1-year period after the last laser treatment, indicating that collagen remodeling occurs over a protracted period of time after laser treatments are performed. Sebum production, as measured by Sebutape scores, was also significantly reduced by the laser treatments, corroborating the hypothesis that the mechanism of this laser involves reduced sebaceous gland activity.

\section{0-nm Erbium Glass Laser}

A single study has also shown efficacy of the 1540-nm erbium glass laser for treatment of acne. After four treatments at 4 -week intervals, a $78 \%$ reduction in acne lesions and decreased skin oiliness was noted in 25 patients. $^{12}$

\section{Intense Pulsed Light}

Unlike a laser, an intense pulsed light source provide a noncoherent (500 to $1200 \mathrm{~nm}$ ) source of intense light that can be modified by filters to provide irradiation with specific wavelengths of light. The use of intense pulsed light for the treatment of acne has been theoretically based on the production of singlet oxygen after photoactivation of porphyrins synthesized and stored by $P$. acnes. ${ }^{13}$ Several studies have utilized intense pulse light sources after topical application of porphyrins, a process discussed later in this article. However, in one study of intense pulsed light, 19 patients with mild to moderate acne were treated using wavelengths between 430 and $1100 \mathrm{~nm}$ at an energy density of $3.5 \mathrm{~J} / \mathrm{cm}^{2}$ and a pulse width of 35 milliseconds. Reductions of $79 \%$ and $74 \%$ were noted in noninflammatory and inflammatory lesions, respectively, 1 month after the final treatment. ${ }^{13}$

\section{Radiofrequency}

A combined radiofrequency and pulsed light device (Aurora AC; Syneron Medical Ltd., Richmond Hill, Ontario, Canada) has recently been used to treat acne. Twice-weekly treatments for 4 weeks resulted in reduction of mean acne lesion counts by $47 \%$ in 32 patients. ${ }^{14}$ The clinical improvement in acne was associated with both reductions in perifollicular inflammation and sebaceous gland areas, and this is presumed to be the mechanism of action whereby this device improves acne. Adverse effects were limited to temporary erythema, tingling, and burning. Another study of 22 patients using a monopolar radiofrequency device (ThermaCool; Thermage, Hayward, CA) showed greater than $75 \%$ reduction in inflammatory acne lesions in more than $90 \%$ of patients after one treatment session. ${ }^{15}$

\section{Low-Intensity Light Treatment and Photodynamic Therapy}

Endogenous coproporphyrins and protoporphyrins produced by $P$. acnes metabolism form the basis of photochemical interactions that result in the destruction of the bacteria. Upon exposure to visible blue, red, or green light, the endogenous porphyrins are excited and in the presence of oxygen generate reactive singlet oxygen species that damage the cell membranes of the bacteria. ${ }^{16,17}$ A recent study of narrowband blue lightemitting diode (409 to $419 \mathrm{~nm}$ ) at $40 \mathrm{~mW} / \mathrm{cm}^{2}$ in 30 patients found significant reduction in inflammatory 

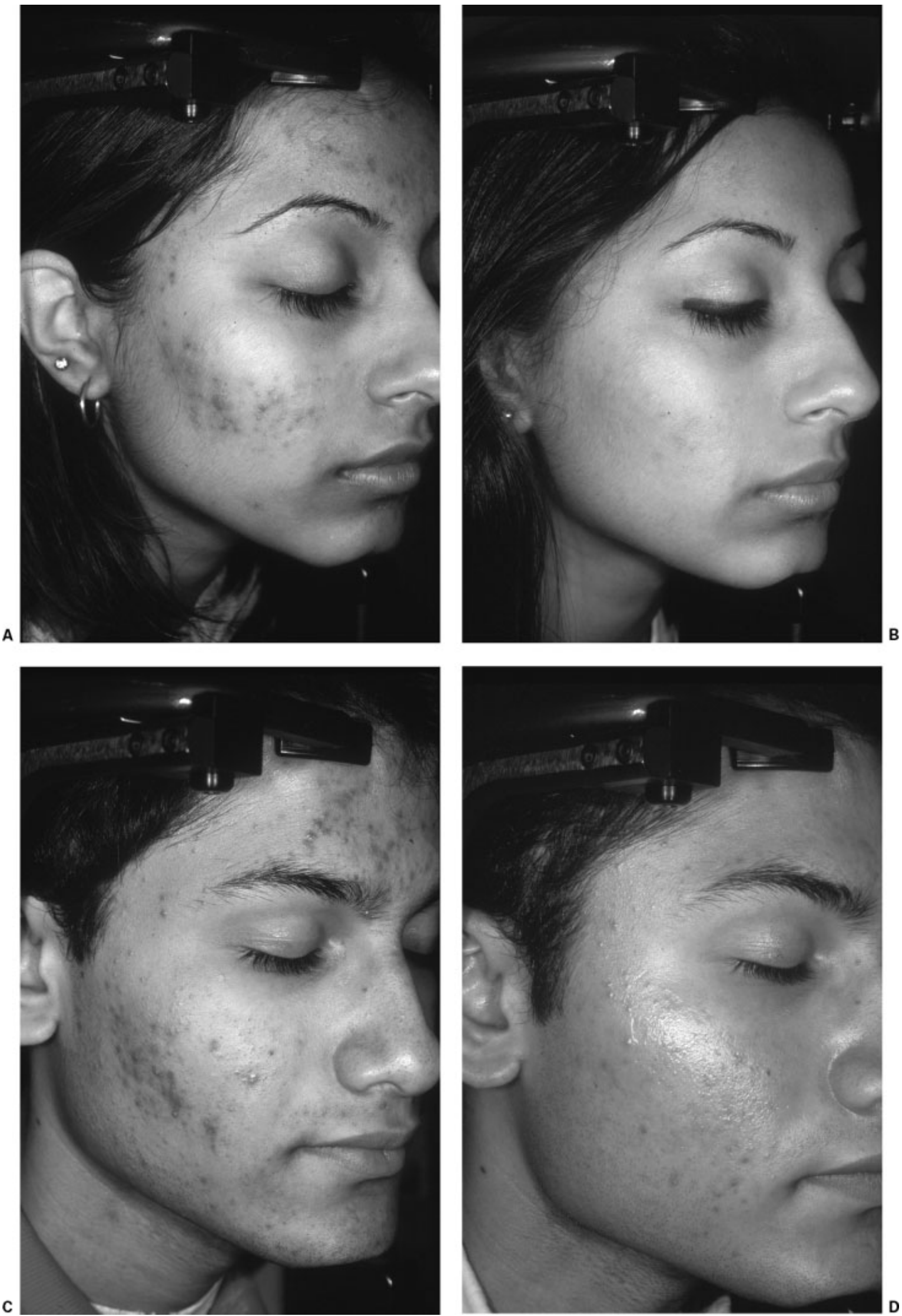

Figure 1 The 1450-nm diode laser provides long-term remission of acne as well as improvement in acne scarring. Comparisons are shown between baseline $(A, C)$ and 12 months after the third and final laser treatment $(B, D)$. 
acne lesion after eight 10 - or 20-minute treatments provided over a 4 -week period. ${ }^{18}$ In another study of 30 patients, a blue light source (ClearLight; Lumenis Inc., Santa Clara, CA) with a peak emission of 407 and $420 \mathrm{~nm}$ was used. Two treatments at $90 \mathrm{~mW} / \mathrm{cm}^{2}$ performed up to 5 weeks apart provided an overall $55 \%$ reduction in acne lesions. ${ }^{19}$

However, the degree of bacterial killing produced by the interaction of endogenous porphyrins with an exogenous light source is limited as only moderate amounts of porphyrins are produced naturally by the bacteria. Enhanced bactericidal activity can be achieved through photodynamic therapy, which involves the addition of the exogenous 5-aminolevulinic acid, a porphyrin precursor that is converted intracellularly to the photoreactive species protoporphyrin IX. ${ }^{20}$ In addition to enhancing bactericidal activity, 5-aminolevulinic acid has also been shown to preferentially accumulate in sebaceous glands and can result in selective damage to sebaceous glands. ${ }^{21}$ Because sebum is actively metabolized into proinflammatory fatty acids by $P$. acnes, the reduction in $P$. acnes combined with reduced sebum production after sebaceous gland damage appears to be the mechanism by which photodynamic therapy utilizing exogenous 5-aminolevulinic acid leads to significant improvement in inflammatory acne.

Typically, 5-aminolevulinic acid is applied to cleaned skin for 3 hours prior to irradiation with blue or red light sources; however, recently, shorter incubation times with 5-aminolevulinic acid followed by irradiation with intense pulsed light or laser have also been shown to be effective and to have significantly reduced adverse effects. ${ }^{22,23} \mathrm{In}$ a study of 14 patients treated with aminolevulinic acid and low-fluence pulsed dye laser, aminolevulinic acid was applied for a short 45-minute incubation followed by treatment with the long-pulse pulsed dye laser (595 nm, 7.0 to $7.5 \mathrm{~J} / \mathrm{cm}^{2}$ fluence). ${ }^{23}$ Complete clearance was achieved in $100 \%$ of patients with a mean of 2.9 treatments required. Moreover, reduction in erythema in acne scars was also seen.

Indocyanine green has also been used for photodynamic therapy of acne. Indocyanine green is a dye that binds albumin and has been shown to be selectively absorbed by sebaceous glands. ${ }^{24}$ After irradiation with a long-pulse diode laser (Cynosure Inc., Westford, MA), a decrease in acne lesions was noted in the treatment area up to 10 months after the last treatment. ${ }^{24}$ In another study, three patients were provided eight treatments utilizing 5-minute topical applications of indocyanine green followed by near-infrared $803-\mathrm{nm}$ diode laser phototherapy at $50 \mathrm{~mW} / \mathrm{cm}^{2}$ for 5 to 10 minutes. Improvements of $80 \%$ were seen 1 month after the last laser treatment. ${ }^{25}$

Although photodynamic therapy has been shown in several studies to be effective for the treatment of inflammatory acne, it has been associated with significant pain and posttreatment erythema, severe phototoxicity, pustular eruptions, and epithelial exfoliation. ${ }^{16-18,20,21}$ The more recent use of methyl aminolevulinate, a methyl ester of 5-aminolevulinic acid, has been shown to be associated with a reduction in both pain and posttreatment adverse effects. ${ }^{26}$

\section{COMPLICATIONS OF LIGHT AND LASER TREATMENT OF ACNE}

In general, as lasers used for acne treatment are nonablative in nature, these treatments have little potential for significant complications. Most patients can expect erythema and edema lasting less than 24 hours. Vascular lasers (KTP and pulsed dye) always have the potential for bruising, particularly in patients taking anticoagulant and antiplatelet agents. Bruises generally resolve in 10 days. Hyperpigmentation develops rarely in darkerpigmented patients undergoing nonablative laser procedures and typically only in those who have not been careful with sun exposure prior to the laser procedure. In our experience with acne patients, this hyperpigmentation is typically mild and resolves completely within a few months. Strict avoidance of the sun both before and during the course of laser treatment must be stressed to all patients. Patients with skin type IV or greater may also benefit from a regimen of pre-laser topical tretinoin and hydroquinone treatment. We are not aware of any development of scarring from these lasers if used properly; however, attention must be paid that any cooling devices that protect the epidermis are being properly used during these procedures so that inadvertent ablation of the epidermis does not occur.

Photodynamic therapy augmented with aminolevulinic acid can be associated with significant treatment-related pain and posttreatment erythema, pustular reactions, and epithelial exfoliation; however, these adverse effects are expected and limited in duration. Severe reactions resulting in blistering can lead to transient hyperpigmentation particularly in skin types IV or greater. Hyperpigmentation can be improved with the use of bleaching creams as well as strict sun avoidance. Avoidance of sunlight and any intense visible light is mandatory for at least 24 hours after aminolevulinic acid exposure, as any light irradiation can induce further porphyrin activation and a resulting phototoxic reaction. As previously discussed, the shorter incubation times that are now being used with the intense pulsed light and laser treatments may reduce the severity of these treatment-related adverse effects.

\section{TREATMENT OF ACNE SCARRING}

Scarring is a common complication of acne for which patients seek laser treatment. Although a comprehensive discussion of the laser treatment of chronic acne scars is 
beyond the scope of this review, a discussion of the laser treatment of acne is not complete without addressing the issue of acne scarring. In general, patients with acne scars must be categorized into those with or without active inflammatory acne.

In the absence of active acne, a variety of ablative and nonablative resurfacing lasers as well as fractional resurfacing have been utilized with moderate success for the treatment of chronic scars. In our experience, traditional ablative resurfacing using the carbon dioxide and erbium:yttrium-aluminum-garnet (Er:YAG) lasers have given way to nonablative resurfacing techniques utilizing lasers such as the 1064-nm neodymium:YAG (Nd:YAG) laser, the 1320-nm Nd:YAG, the 1450-nm diode laser, and pulsed dye lasers, among others. ${ }^{27-30}$

More recently, fractional photothermolysis (Fraxel; Reliant Technologies, Mountainview, CA), has been introduced. These units utilize a 1520 - to 1560 -nm erbium fiber laser to create an array of numerous microscopic thermal wounds to achieve skin rejuvenation without the side effects associated with ablative lasers. ${ }^{31}$ The efficacy of fractional photothermolysis for acne scars has been reported in one study ${ }^{32}$; however, based on our own experience, this technology has rapidly become our first choice in the treatment of acne scarring, as it appears significantly more effective than other nonablative resurfacing techniques.

In the presence of inflammatory acne, the primary treatment should be directed at long-term remission of inflammatory acne to cease the formation of new scars. For such patients, treatment considerations should include a variety of medical treatments, including oral isotretinoin for severe cases. Moreover, consideration should be given to lasers that have documented efficacy both for inflammatory acne as well as acne scarring. These include the pulsed dye laser, the 1320-nm Nd:YAG laser, as well as the $1450-n m$ diode laser.

The pulsed dye laser has been shown to provide significant improvement in both acne (see discussion above) and acne scarring. In one study, 22 patients with erythematous or hypertrophic facial acne scars were enrolled in a split-face study. ${ }^{4}$ Significant clinical improvement was observed in laser-irradiated acne scars after one or two treatments with the $585-\mathrm{nm}$ pulsed dye laser. Given the efficacy of this laser in treating both inflammatory acne as well as acne scars, it should be considered in patients with evidence of both processes.

Perhaps the best-studied laser for the treatment of patients with both inflammatory acne and acne scars is the 1450-nm diode laser. We have found both long-term reductions in inflammatory acne lesions and clinically and statistically significant improvements in acne scars. ${ }^{11}$ The improvement in acne scars develops over several months after laser treatments end, so patients should be told to expect gradual improvement over time.

\section{LASER TREATMENT OF RELATED CONDITIONS}

\section{Pseudofolliculitis Barbae}

Pseudofolliculitis barbae is a common skin disorder of the beard area characterized by inflammatory follicular papules due to emerging hair shafts reentering the epidermis. Laser treatment of this condition tends to concentrate on hair removal techniques to target the underlying follicular structure. Initially, the Q-switched $\mathrm{Nd}$ :YAG laser was used in conjunction with topical carbon suspension to improve follicular targeting by the laser. ${ }^{33}$ Subsequently, Kauvar reported greater than $50 \%$ improvement in pseudofolliculitis barbae in 10 patients after three treatments with the 810 -nm diode laser (pulse duration 20 to 30 milliseconds, fluence 30 to $40 \mathrm{~J} / \mathrm{cm}^{2}$, treatment interval 6 to 8 weeks). A $50 \%$ reduction in hair density was noted in all subjects. ${ }^{34}$

As most patients with pseudofolliculitis barbae have dark skin complexions, the development of longpulse lasers has improved the safety profile of hair removal in these patients. In one study, a reduction in lesion count from 6.95 on the adjacent untreated control site to 1.0 on the site treated with a single treatment of the long-pulse Nd:YAG laser was observed. ${ }^{35}$ In a subsequent split-neck study, the number of papules was counted 1 month after a single treatment with the long-pulse Nd:YAG and compared with the untreated side. Mean postoperative papule counts were 11.6 and 30.1 for the treated and untreated sides, respectively. ${ }^{36}$ A $75 \%$ reduction in lesion count was reported in $13 \mathrm{pa-}$ tients undergoing three treatments at 2-week intervals using a modified superlong-pulse 810 -nm diode laser (Palomar Medical, Burlington, MA). ${ }^{37}$

For patients with refractory pseudofolliculitis barbae, hair removal laser should be considered as a treatment option. The expected reduction in beard area hair counts, which may be permanent, should be discussed with patients. In our experience, some patients with moderate refractory pseudofolliculitis barbae have experienced clinically significant and sustained improvements with the 1450-nm diode laser (Smoothbeam; Candela Corp., Wayland, MA). As this laser does not cause reduction in hair density, it should be considered for the treatment of male patients with pseudofolliculitis barbae.

\section{Acne Keloidalis Nuchae}

Acne keloidalis nuchae is a chronic inflammatory process affecting the hair follicles of the occipital scalp. Small follicular pustules develop into keloid-like scars. The disease is often progressive and deforming as new crops of lesions continue to form, leaving behind keloidal papules. Traditional treatments, including oral and topical antibiotics and intralesional corticosteroids, are used 
commonly with variable success. In severe cases, surgical excision or carbon dioxide laser ablation followed by secondary-intention healing have been tried. ${ }^{38}$

The use of hair removal lasers in acne keloidalis nuchae has been reported. One case series reported clinical improvement in three patients treated with the ruby laser. ${ }^{39}$ This improvement was seen in conjunction with decreased hair growth in the affected area. Most recently, a small study of two patients demonstrated 90 to $95 \%$ improvement in lesions after four treatments with the diode laser. Although hair removal lasers would be expected to significantly improve this condition, the potential for prolonged or permanent alopecia in the occipital scalp makes this an undesirable option for most patients and should be considered in only the most refractory cases.

\section{Conclusion}

Laser- and light-based acne treatments have become increasingly accepted as alternatives to traditional medical treatments for inflammatory acne due to the high degree of clinical efficacy and minimal adverse effects. The limited number of office-based treatments required is particularly attractive to patients who have difficulty complying with complex long-term regimens involving combinations of topical and oral agents. These devices also offer an option for those patients who have moderate to severe acne but are concerned about oral medications because of potential adverse effects. Long-term improvements up to 1 year have been documented with laser treatments. ${ }^{11}$ The reported clinical efficacy compares favorably with that achieved with oral isotretinoin without the risk of systemic toxicity. In addition, in light of recent regulations regarding mandatory registration for all patients taking oral isotretinoin, laser- and lightbased treatments have become an increasingly attractive alternative for both practitioners as well as patients with moderate to severe acne who prefer not to navigate the cumbersome new regulatory system. Moreover, significant concomitant improvement in acne scarring provided by lasers is an advantage that no medical treatment provides. Future advances in laser technology as well as continued clinical refinements will likely improve both the efficacy and safety of lasers for the treatment of acne vulgaris and will likely make laser- and light-based treatments an even more attractive and cost-effective option for patients with acne and acne scarring.

\section{REFERENCES}

1. Oberemok SS, Shalita AR. Acne vulgaris, I: pathogenesis and diagnosis. Cutis 2002;70:101-105

2. Baugh WP, Kacuba WD. Nonablative phototherapy for acne vulgaris using the KTP $532 \mathrm{~nm}$ laser. Dermatol Surg 2005; 31:1290-1296
3. Lee MC. Aura $532 \mathrm{~nm}$ laser for acne vulgaris-3-year experience. Presented at: 2003 ASDS-ACMMSCO Combined Annual Meeting; October 9-12, 2003; New Orleans, LA

4. Alster TS, McMeekin TO. Improvement of facial acne scars by the $585 \mathrm{~nm}$ flashlamp-pumped pulsed dye laser. J Am Acad Dermatol 1996;35:79-81

5. Seaton ED, Mouser PE, Charakida A, Alam S, Seldon PE, Chu AC. Investigation of the mechanism of action of nonablative pulsed-dye laser therapy in photorejuvenation and inflammatory acne vulgaris. Br J Dermatol 2006;155: 748-755

6. Seaton ED, Charakida A, Mouser PE, Grace I, Clement RM, Chu AC. Pulsed-dye laser treatment for inflammatory acne vulgaris: randomized controlled trial. Lancet 2003;362: 1347-1352

7. Orringer JS, Kang S, Hamilton T, et al. Treatment of acne vulgaris with a pulsed dye laser: a randomized controlled trial. JAMA 2004;291:2834-2839

8. Glaich AS, Friedman PM, Jih MH, Goldberg LH. Treatment of inflammatory facial acne vulgaris with combination 595-nm pulsed-dye laser with dynamic-cooling-device and 1,450-nm diode laser. Lasers Surg Med 2006;38:177-180

9. Paithankar DY, Ross EV, Saleh BA, Blair MA, Graham BS. Acne treatment with a $1,450 \mathrm{~nm}$ wavelength laser and cryogen spray cooling. Lasers Surg Med 2002;31:106-114

10. Friedman PM, Jih MH, Kimyai-Asadi A, Goldberg LH. Treatment of inflammatory facial acne vulgaris with the 1450-nm diode laser: a pilot study. Dermatol Surg 2004;30: 147-151

11. Jih MH, Friedman PM, Goldberg LH, Robles M, Glaich AS, Kimyai-Asadi A. The 1450-nm diode laser for facial inflammatory acne vulgaris: dose-response and 12-month follow-up study. J Am Acad Dermatol 2006;55:80-87

12. Boineau D, Angel S, Auffret N, Dahan S, Mordon S. Treatment of active acne with an erbium glass (1.54 micron) laser. Lasers Surg Med 2004;16:S55

13. Elman M, Lask G. The role of pulsed light and heat energy (LHE) in acne clearance. J Cosmet Laser Ther 2004;6: 91-95

14. Prieto VG, Zhang PS, Sadick NS. Evaluation of pulsed light and radiofrequency combined for the treatment of acne vulgaris with histologic analysis of facial skin biopsies. J Cosmet Laser Ther 2005;7:63-68

15. Ruiz-Esparza J, Gomez JB. Nonablative radiofrequency for active acne vulgaris: the use of deep dermal heat in the treatment of moderate to severe active acne vulgaris (thermotherapy): a report of 22 patients. Dermatol Surg 2003;29:333-339

16. Ashkenazi H, Malik Z, Harth Y, Nitzan Y. Eradication of Propionibacterium acnes by its endogenic porphyrins after illumination with high intensity blue light. FEMS Immunol Med Microbiol 2003;35:17-24

17. Papageorgiou P, Katasambas A, Chu A. Phototherapy with blue $(415 \mathrm{~nm})$ and red $(660 \mathrm{~nm})$ light in the treatment of acne vulgaris. Br J Dermatol 2000;142:973-978

18. Morton CA, Scholefield RD, Whitehurst C, Birch J. An open study to determine the efficacy of blue light in the treatment of mild to moderate acne. J Dermatolog Treat 2005;16:219-223

19. Kawada A, Aragne Y, Kameyama H, Sangen Y, Tezuka T. Acne phototherapy with high-intensity, enhanced, narrowband, blue light source: an open study and in vitro investigation. J Dermatol Sci 2002;30:129-135 
20. Itoh Y, Ninimiya Y, Tajima S, Ishibashi A. Photodynamic therapy of acne vulgaris with topical delta-aminolevulinic acid and incoherent light in Japanese patients. Br J Dermatol 2001;144:575-579

21. Hongcharu W, Taylor CR, Chang Y, Aghassi D, Suthamjariya K, Anderson RR. Topical ALA-photodyanimc therapy for the treatment of acne vulgaris. J Invest Dermatol 2000; 115:183-192

22. Rojanamatin J, Choawawanich P. Treatment of inflammatory facial acne vulgaris with intense pulsed light and short contact of topical 5-aminolevulinic acid: a pilot study. Dermatol Surg 2006;32:991-996

23. Alexiades-Armenakas M. Long-pulsed dye laser-mediated photodynamic therapy combined with topical therapy for mild to severe comedonal, inflammatory or cystic acne. J Drugs Dermatol 2006;5:45-55

24. Lloyd JR, Mirkov M. Selective photothermolysis of the sebaceous glands for acne treatment. Lasers Surg Med 2002; 31:115-120

25. Genina EA, Bachkaov AN, Simonenko GV, Odoevskaya OD, Tuchin VV, Altshuler GB. Low intensity indocyaninegreen laser phototherapy of acne vulgaris: pilot study. J Biomed Opt 2004;9:828-834

26. Wiegell SR, Wulf HC. Photodynamic therapy of acne vulgaris using 5-aminolevulinic acid versus methyl aminolevulinate. J Am Acad Dermatol 2006;54:647-651

27. Tanzi EL, Lupton JR, Alster TS. Lasers in dermatology: four decades of progress. J Am Acad Dermatol 2003;49:1-31

28. Friedman PM, Jih MH, Skover GR, Payonk GS, KimyaiAsadi , Geronemus RG. Treatment of atrophic facial acne scars with the 1064-nm Q-switched Nd:YAG laser: sixmonth follow-up study. Arch Dermatol 2004;140:1337- 1341

29. Yaghmai D, Garden JM, Bakus AD, Massa MC. Comparison of a $1,064 \mathrm{~nm}$ laser and a 1,320 nm laser for the nonablative treatment of acne scars. Dermatol Surg 2005; 31:903-909
30. Fulchiero GJ Jr, Parham-Vetter PC, Obagi S. Subsiciosn and 1320-nm Nd:YAG nonablative laser resurfacing for the treatment of acne scars; a simultaneous split-face single patient trial. Dermatol Surg 2004;30:1356-1359

31. Manstein D, Herron GS, Sink RK, Tanner H, Anderson RR. Fractional photothermolysis: a new concept for cutaneous remodeling using microscopic patterns of thermal injury. Lasers Surg Med 2004;34:426-438

32. Hasegawa T, Matsukura T, Mizuno Y, Suga Y, Ogawa H, Ikeda S. Clinical trial of a laser device called fractional photothermolysis system for acne scars. J Dermatol 2006; 33:623-627

33. Rogers CJ, Glaser DA. Treatment of pseudofolliculitis barbae using the Q-switched Nd:YAG laser with topical carbon suspension. Dermatol Surg 2000;26:737-742

34. Kauvar AN. Treatment of pseudofolliculitis with a pulsed infrared laser. Arch Dermatol 2000;136:1343-1346

35. Ross EV, Cooke LM, Timko AL, Overstreet KA, Graham BS, Barnette DJ. Treatment of pseudofolliculitis barbae in skin types IV, V, and VI with a long-pulsed neodymium: yttrium aluminum garnet laser. J Am Acad Dermatol 2002; 47:263-270

36. Ross EV, Cooke LM, Overstreet KA, Buttolph GD, Blair MA. Treatment of pseudofolliculitis barbae in very dark skin with a long pulse Nd:YAG laser. J Natl Med Assoc 2002;94: 888-893

37. Smith EP, Winstanley D, Ross EV. Modified superlong pulse $810 \mathrm{~nm}$ diode laser in the treatment of pseudofolliculitis barbae in skin types V and VI. Dermatol Surg 2005;31:297301

38. Kantor GR, Ratz JL, Wheeland RG. Treatment of acne keloidalis nuchae with carbon dioxide laser. J Am Acad Dermatol 1986;14:263-267

39. Chui CT, Berger TG, Price VH, Zachary CB. Recalcitrant scarring follicular disorders treated by laser-assisted hair removal: a preliminary report. Dermatol Surg 1999;25:34-37 